\title{
Evaluation of electric nets as means to sample mosquito vectors host-seeking on humans and primates
}

Frances Hawkes ${ }^{1 *}$, Benny Obrain Manin², Sui Han Ng${ }^{2}$, Stephen J Torr ${ }^{3}$, Chris Drakeley ${ }^{4}$, Tock H Chua ${ }^{2}$ and Heather M. Ferguson ${ }^{5}$

\begin{abstract}
Background: Plasmodium knowlesi is found in macaques and is the only major zoonotic malaria to affect humans. Transmission of $P$. knowlesi between people and macaques depends on the host species preferences and feeding behavior of mosquito vectors. However, these behaviours are difficult to measure due to the lack of standardized methods for sampling potential vectors attracted to different host species. This study evaluated electrocuting net traps as a safe, standardised method for sampling P. knowlesi vectors attracted to human and macaque hosts. Field experiments were conducted within a major focus on $P$. knowlesi transmission in Malaysian Borneo to compare the performance of human (HENET) or macaque (MENET) odour-baited electrocuting nets, human landing catches (HLC) and monkey-baited traps (MBT) for sampling mosquitoes. The abundance and diversity of Anopheles sampled by different methods were compared over 40 nights, with a focus on the P. knowlesi vector Anopheles balabancensis.

Results: HLC caught more An. balabacensis than any other method (3.6 per night). In contrast, no An. balabacensis were collected in MBT collections, which generally performed poorly for all mosquito taxa. Anopheles vector species including An. balabacensis were sampled in both HENET and MENET collections, but at a mean abundance of less than 1 per night. There was no difference between HENET and MENET in the overall abundance $(P=0.05)$ or proportion $(P=0.7)$ of An. balabacensis. The estimated diversity of Anopheles species was marginally higher in electrocuting net than HLC collections, and similar in collections made with humans or monkey hosts.

Conclusions: Host-baited electrocuting nets had moderate success for sampling known zoonotic malaria vectors. The primary vector An. balabacensis was collected with electrocuting nets baited both with humans and macaques, but at a considerably lower density than the HLC standard. However, electrocuting nets were considerably more successful than monkey-baited traps and representatively characterised anopheline species diversity. Consequently, their use allows inferences about relative mosquito attraction to be meaningfully interpreted while eliminating confounding factors due to trapping method. On this basis, electrocuting net traps should be considered as a useful standardised method for investigating vector contact with humans and wildlife reservoirs.
\end{abstract}

Keywords: Anopheles balabacensis, Plasmodium knowlesi, Electrocuting traps, Zoonosis, Surveillance, Macaque, Trapping bias

\footnotetext{
* Correspondence: f.m.hawkes@greenwich.ac.uk

Frances Hawkes and Benny Obrain Manin are equal first authors

Tock H Chua and Heather M Ferguson are equal senior authors

${ }^{1}$ Agriculture Health \& Environment Department, Natural Resources Institute,

University of Greenwich, Central Avenue, Chatham, Kent ME4 4TB, UK

Full list of author information is available at the end of the article
}

(c) The Author(s). 2017 Open Access This article is distributed under the terms of the Creative Commons Attribution 4.0 International License (http://creativecommons.org/licenses/by/4.0/), which permits unrestricted use, distribution, and reproduction in any medium, provided you give appropriate credit to the original author(s) and the source, provide a link to the Creative Commons license, and indicate if changes were made. The Creative Commons Public Domain Dedication waiver (http://creativecommons.org/publicdomain/zero/1.0/) applies to the data made available in this article, unless otherwise stated. 


\section{Background}

Control of vector-borne diseases requires identification of when and where people are exposed to bites from insect vectors, and which vector species are responsible for transmission. Previously, the human landing catch (HLC) has been viewed as the "gold standard" approach for assessing human exposure to mosquito vectors [1]. This procedure requires participants to expose part of their body to mosquitoes, usually the lower leg, and collect all insects that land upon them. As this method gives a direct estimate of the number and infection status of mosquitoes drawn to a human, it is widely used in vector surveillance, research and intervention evaluation. However, this method has a number of limitations. The most serious is that it requires technicians to collect mosquitoes landing on their bare skin and so exposes them to a range of vector-borne diseases, including malaria, dengue, chikungunya virus, filariasis and viral encephalitis, many of which have no prophylactic and/or limited treatment options [2]. The emergence [3] and spread [4] of drug-resistant $P$. falciparum malaria in mainland Asia and the lack of effective prophylaxis for other mosquito-borne pathogens such as dengue and Zika virus make routine use of HLCs particularly problematic in Southeast Asian settings.

Another limitation of HLCs is that, by definition, they can only be performed by humans. In the context of zoonotic disease, there is a need for comparative sampling of mosquitoes biting on both people and other potential animal reservoir species. To date, there are few methods available for comparing mosquito biting rates on people and other animals; and to our knowledge none have been standardised to provide direct comparison with HLC data. Researchers have investigated a range of different sampling methods to collect mosquitoes attracted to animal hosts, including comparisons between HLC and baited net traps for monkeys [5-7]. Although useful for qualitative comparison, inherent differences in the biases and efficiency between sampling methods [8] make it difficult to determine if observed differences between host types are due to their differential attractiveness, or the trapping methods themselves. Accurate and ethically appropriate vector sampling methods for zoonoses thus require trapping approaches that are (i) suitable for use with either humans or other animals as host baits; (ii) limit or eliminate human (and animal) participants' exposure to vector-borne diseases; and (iii) standardise inherent biases, irrespective of bait animal.

The need to develop standardised methods for measuring mosquito biting has particular relevance for the malaria parasite Plasmodium knowlesi. Of all malaria species of public health importance, $P$. knowlesi is the only species with a significant wildlife reservoir, specifically long- and pig-tailed macaques (Macaca species) [9].
In 2004, the first large focus of human infection with this zoonotic simian malaria parasite was reported in rural Sarawak, Malaysian Borneo [10]. Since then, this expanding zoonosis has been reported in all Southeast Asian countries with the exception of Laos PDR [11]. Malaysian Borneo is recognized as the epicentre of human cases, with $P$. knowlesi now responsible for the majority of local malaria infections in people [12].

Anopheline mosquitoes of the Leucosphyrus group have been implicated as potential vectors in the cross-species transfer of P. knowlesi from macaques to humans [11], although other anopheline vectors may be as yet unidentified. Within this group, the vector species responsible for transmission varies geographically. The strongly anthropophilic species Anopheles dirus transmits P. knowlesi from macaques to humans in Cambodia, Vietnam, China and Thailand [13], while in the west Malaysian state of Pahang, An. cracens is considered to be the main vector [7]. However, in east Malaysian states, two vectors have been identified: An. latens in Sarawak [14] and An. balabacensis in Sabah [15]. The vector species involved in $P$. knowlesi transmission are confirmed to feed on humans, although the degree to which they feed on macaque reservoir species and other wildlife is unclear due to the paucity of available sampling methods. Acquiring such data on relative host preferences of zoonotic malaria vectors is vital for understanding human exposure risk and targeting control measures.

Electrocuting traps may offer a solution to some of the issues associated with traditional mosquito trapping methods [16]. These devices were originally developed to quantify the numbers of tsetse flies attracted to humans and wildlife hosts (warthogs) by placing electrocuting nets in an incomplete ring around the host species [17]. The electrocuting net is effectively invisible to tsetse and hence as they approach the host, tsetse inadvertently collide with it and are either killed or stunned; with the number caught outside and inside indicating their abundance and feeding success. Further variants of these traps have been developed and used to investigate aspects of mosquito behaviour including flight [18] and oviposition behaviour [19]. Early electrocuting traps were often based on placing an electrocuting surface next to the point where host odour was released via a pipe (e.g. electric grids [20]), and important subtleties in the behavioural responses of African malaria vectors to odours from humans and livestock cattle have been demonstrated using this approach [21]. Recently developed alternatives work as a barrier placed immediately around a host (e.g. "Mosquito Electrocuting Traps" [22]) and have been shown to be reliable proxies for HLC in some situations [23]. However, the use of such devices to measure mosquito attraction to wildlife has not yet been investigated, nor has their efficacy with more exophilic South East Asian vectors. 
Here, we assessed the feasibility of using electrocuting traps as a standardised method to quantify mosquito vector biting rates on humans and macaques, and identify which species have potential to transmit $P$. knowlesi between these hosts. We first tested the suitability of electrocuting nets as an alternative to existing reference trapping methods used for sampling mosquitoes attracted to humans (HLC) and macaques (monkey-baited trap). Secondly, catches from human- and macaquebaited electrocuting nets were compared to determine if and how the mosquito fauna attracted to these different host species vary. Results are discussed with a view to providing new ethically appropriate and scientifically robust methods for vector research and surveillance of zoonotic vector-borne diseases.

\section{Methods}

\section{Study site}

The study was conducted in an area of newly-cleared secondary rainforest outside the village of Tajau Laut $\left(6^{\circ} 57^{\prime}\right.$ $\left.44.5^{\prime \prime} \mathrm{N}, 116^{\circ} 48^{\prime} 56.3^{\prime \prime} \mathrm{E}\right)$ in the Kudat district of Sabah, the northern-most state of Malaysian Borneo. The region has a high prevalence of P. knowlesi; $76 \%$ of 455 malaria patient samples taken from Kudat District Hospital between 2009 and 2011 were found to be positive for P. knowlesi mono-infection by PCR [24]. This village was selected as the study site due to the occurrence of confirmed local cases of human infection with P. knowlesi (M. Grigg, pers. comm./Kudat District Hospital) and reports from residents indicating that both long-tailed (Macaca fascicularis) and pig-tailed (Macaca nemestrina) macaques were frequently seen near the village. Additionally, the presence of Leucosphyrus group mosquitoes was confirmed by preliminary HLCs. Experiments were conducted between November 2013 and January 2014, coinciding with the northeast monsoon season of high rainfall and corresponding period of highest local mosquito densities.

\section{Trapping methods \\ Human landing catch (HLC)}

Human landing catches were performed by trained collectors working in pairs. Glass tubes were placed over mosquitoes as they alighted on a collector's exposed leg, and then the tube was sealed with cotton wool. Collections were carried out between 18:00 and 06:00 h each day. During each hour, mosquitoes were collected for $45 \mathrm{~min}$, followed by a $15 \mathrm{~min}$ rest break for the collectors, and stored in bags labelled by hour. Members of the collection team rotated, so that only one exposed their leg during each hour, the other helping to catch the mosquitoes.

\section{Monkey-baited trap (MBT)}

In previous studies of primate malaria vectors, monkeybaited traps have been used as the reference method to sample mosquitoes attracted to macaques [5-7]. We modified this approach slightly as follows: two juvenile longtailed macaques were placed inside a steel cage measuring $1.8 \times 2.0 \times 2.0 \mathrm{~m}$ and fitted with wire mosquito mesh $(2 \times 2 \mathrm{~mm})$ to prevent entry of mosquitoes and predators (see Fig. 1a, Ethics approval and Additional file 1 for further details of primate use in research). A metal frame, $2.55 \times 2.75 \times 2.75 \mathrm{~m}$ (i.e. $0.75 \mathrm{~m}$ larger than the cage in all dimensions), was erected over the cage and a large, untreated mosquito net was suspended from it, encompassing the cage on all sides. A small opening along the bottom edge at either end of the net was made by rolling up the net from the ground to a height of $\sim 0.4 \mathrm{~m}$ (Fig. 1b). Hostseeking mosquitoes were therefore free to enter the net via the opening but were prevented from feeding by the cage's internal mesh, which protected macaques from mosquito bites. This internal protective net has not been previously incorporated into MBT designs and was a requirement of the ethics approvals granted to work with primates. Mosquitoes attempting to exit were either trapped between the cage and outer net, or could leave via the $0.4 \mathrm{~m}$ floor opening. At the end of each night's experiments, a collector would enter the mosquito net, close the openings, and collect mosquitoes resting on the inside of the net using glass tubes and cotton wool. These samples therefore represented a whole night's catch and were not distinguished by hour of collection.

\section{Electrocuting nets}

Electrocuting nets ( $1 \mathrm{~m}$ high; $0.5 \mathrm{~m}$ wide) consisted of a sheet of fine black polyester net sandwiched between two banks of vertical copper wires, $0.2 \mathrm{~mm}$ in diameter, $5 \mathrm{~mm}$ apart, $8 \mathrm{~mm}$ from each side of the net and spraypainted black (Fig. 1c). The net and wires are intended to be invisible to nocturnal flying insects. Alternate wires were earthed or charged by a transformer with a DC input $(12 \mathrm{~V} ; 3 \mathrm{~A})$ and an output of $50 \mathrm{kV}$, pulsing at $\sim 70 \mathrm{~Hz}$. Insects killed or stunned after colliding with the grids were collected in trays placed under either side of the electrocuting net $(0.7 \mathrm{~m}$ long; $0.5 \mathrm{~m}$ wide; $0.15 \mathrm{~m}$ deep), each containing $\sim 5 \mathrm{l}$ water and $15 \mathrm{ml}$ dissolved detergent. This design is identical to that developed for tsetse fly research [17], with the exception that vertical wires were spaced $5 \mathrm{~mm}$ apart, as opposed to the $8 \mathrm{~mm}$ spacing used for tsetse. Previous optimisation experiments in the field showed that a $5 \mathrm{~mm}$ spacing was better-suited to local mosquito fauna (data not shown). A marquee erected over electrocuting nets protected them from rain and prevented collection trays from flooding.

Single electrocuting nets were baited with natural host odours from either (i) two humans, or (ii) two macaques (Fig. 1d). Human volunteers were housed inside a tent measuring $2.0 \times 2.0 \times 2.0 \mathrm{~m}$ constructed from plasticcoated canvas; macaques were housed in a cage as 

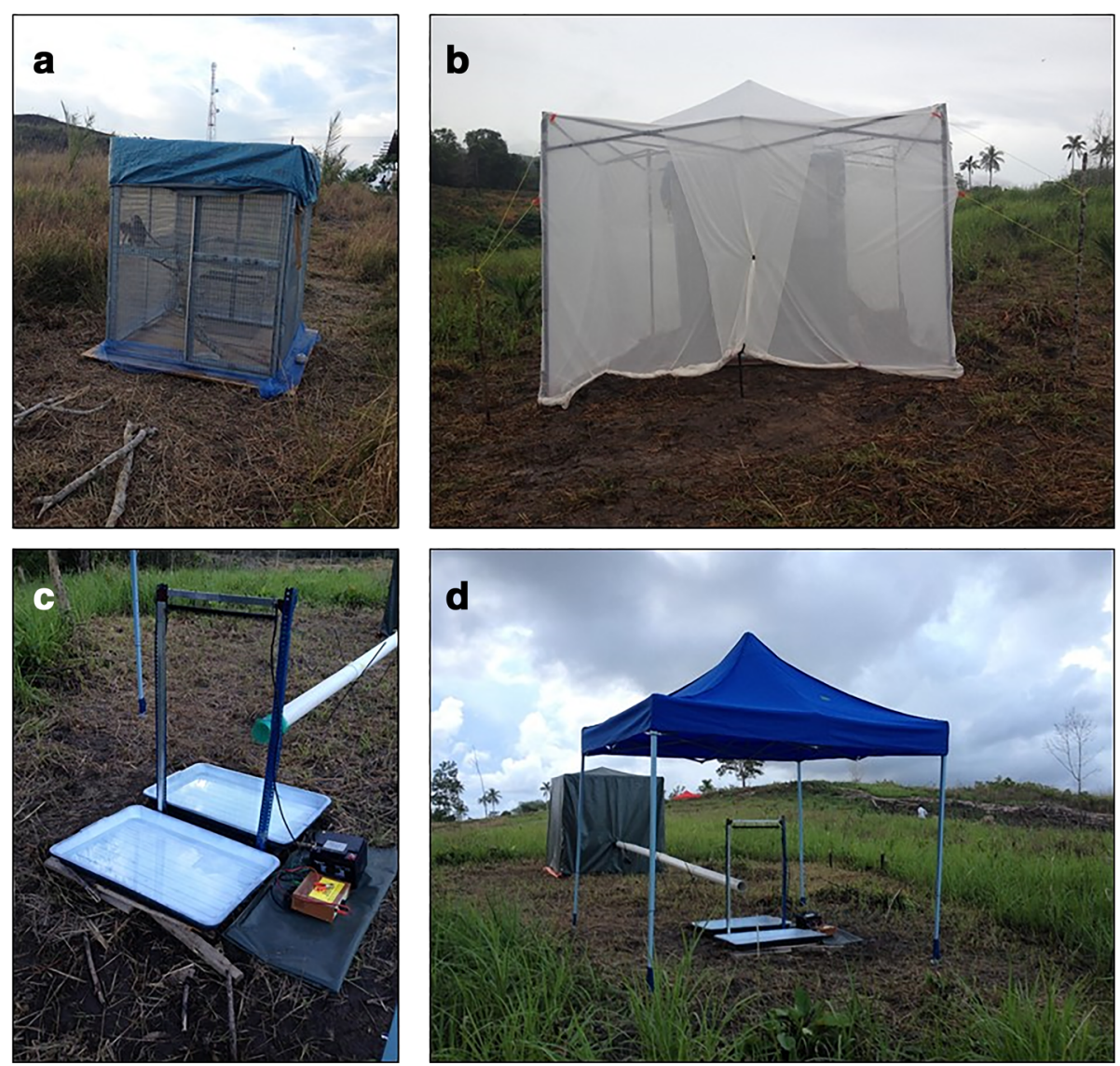

Fig. 1 Trapping devices. a Ethically compliant cage for two juvenile macaques (see Additional file 1). b Monkey-baited trap (MBT): the macaque cage is enclosed in a netted frame, with $\sim 0.4 \mathrm{~m}$ of netting rolled up at either end of the net; host-seeking mosquitoes enter the net in search of macaques and are collected from inside the net. c Electrocuting net, water collection trays, battery and transformer, with pipe leading from tent. d Electrocuting net trap: either two humans (HENET) or two macaques (MENET) are housed in the tent, their odours then vented via the tube to an array of charged wires; host-seeking mosquitoes are electrocuted when investigating the host odour and fall into wet collection trays beneath the net

described for MBTs, the tent then constructed around this. A coaxial fan $(12 \mathrm{~V} ; 0.38 \mathrm{~A}$; maximum airflow $\sim 2000 \mathrm{l} / \mathrm{min}$ ) drew odour-laden air from the tent down a length of plastic tubing (10 m long, $0.1 \mathrm{~m}$ diameter) to a net-covered outlet. The outlet was positioned to release host odour towards the centre of an electrocuting net, with the outlet positioned $\sim 30 \mathrm{~cm}$ away from the center of the vertical wire array. As with MBTs, mosquito specimens were collected once at the end of the collection period and retrieved from the collecting trays using fine forceps. The grids themselves were also inspected for any mosquitoes attached to the wires.

\section{Study design}

A comparative evaluation of human-baited (HENET) and macaque-baited (MENET) electrocuting nets against existing trapping methods for these hosts (HLC and MBT, respectively) was carried out between November 2013 and January 2014 in Tajau Laut, Kudat, Sabah. Experiments followed a randomised $4 \times 4$ Latin square design of traps $\times$ sites $\times$ nights and took place over 40 nights (10 replicates), sampling within replicates occurring on 4 consecutive nights. Sampling sites were between 100 and $150 \mathrm{~m}$ apart and collections were conducted between 18:00 h and 06:00 h. Paired HLC collectors and paired macaques were selected from a pool (8 men; 6 macaques) and rotated randomly throughout the experiment between HLC and HENET, and MBT and MENET, respectively.

\section{Mosquito identification}

After collection, HLC and MBT specimens were returned to the field lab and stored in sealed tubes with silica gel at $-15{ }^{\circ} \mathrm{C}$, with samples transferred to $-80{ }^{\circ} \mathrm{C}$ at the end of each replicate. Wet specimens from electrocuting net collection trays were stored in $70 \%$ ethanol. Mosquitoes from each trap were then counted and identified morphologically to genus and then species where possible using a dissecting microscope according to the keys of Sallum et al. $[25,26]$ for anopheline species, and Rattanarithikul et al. [27] for culicine species. 


\section{Plasmodium detection}

All 584 Anopheles females collected during the study were screened for malaria parasites. Each Anopheles individual was cut into two parts (abdomen, and thorax plus head) and the total DNA was extracted from each section using DTAB-CTAB method [28]. Malaria parasites were detected from the specimens using a nested PCR assay as described by Singh et al. [29]. For Plasmodium positive specimens, another nested PCR assay was performed to determine the species using nine speciesspecific primers (Additional file 2: Table S1). Another set of nested PCR assays were performed separately on the same time interval as internal control by targeting the Anopheles DNA cox 2 gene. All three nested PCR assays were performed with $25 \mu$ final volume in the first and second PCR reactions consisting of $5.0 \mu \mathrm{l}$ of $5 \times$ PCR buffer (Promega, Singapore), $0.5 \mu \mathrm{l}$ of dNTPs (10 mM) mixture (Promega), $3.0 \mu \mathrm{l}$ of $25 \mathrm{mM} \mathrm{MgCl}_{2}$ (Promega), $1.0 \mu \mathrm{l}$ each of $10 \mu \mathrm{M}$ forward and reverse primers, $0.3 \mu \mathrm{l}$ of Tag DNA polymerase $(5 \mathrm{U} / \mu \mathrm{l}), 2.0 \mu \mathrm{l}$ of DNA template and sterile $\mathrm{dH}_{2} \mathrm{O}$ up to $25 \mu \mathrm{l}$ final volume. After completion of the first PCR, $2.0 \mu \mathrm{l}$ of PCR product was used as DNA template in the second PCR. The PCR conditions were: an initial denaturation at $95{ }^{\circ} \mathrm{C}$ for $5 \mathrm{~min}$, followed by 35 cycles of $94^{\circ} \mathrm{C}$ for $1 \mathrm{~min}$, annealing for $1 \mathrm{~min}$ and $72{ }^{\circ} \mathrm{C}$ for $1 \mathrm{~min}$, and a final extension at $72{ }^{\circ} \mathrm{C}$ for $5 \mathrm{~min}$. The annealing temperature was set based on the optimum temperature of the primer with range from $50{ }^{\circ} \mathrm{C}$ to $66{ }^{\circ} \mathrm{C}$ (Additional file 2: Table S1).

\section{Data analysis}

Statistical analyses were performed in $\mathrm{R}$ version 3.3.1, with the packages boot, glmmADMB, lme4, MASS and multcomp [30-35].

\section{Mosquito diversity}

Three species diversity indices were calculated for each trap type based on all mosquito fauna collected. Species richness $(R)$, represented by a count of the total number of different species collected by each trap type, was accompanied by the Gini-Simpson's Diversity Index (1 $D)$, where:

$$
1-D=1-\frac{\sum n_{i}\left(n_{i}-1\right)}{N(N-1)}
$$

the $95 \%$ confidence limit of which is:

$$
\pm 2 \sqrt{\frac{\sum\left(\frac{n_{i}}{N}\right)^{2}-\left(\sum\left(\frac{n_{i}}{N}\right)^{2}\right)^{2}}{N(N-1)}}
$$

Herein, $n_{i}$ is the abundance of species $i$, and $N$ is the total number of individuals in a sample. The relative abundance of different species in each trap was measured using Simpson's Index of Evenness $(E)$, using the formula:

$$
E=\frac{D}{D_{\max }}
$$

in which $D_{\max }$ is the highest value of $D$, for the given number of species and sample size [36].

\section{Anopheles abundance}

Negative binomial generalized linear mixed models (GLMMs) were used to analyse variation in Anopheles species abundance between different collection methods [37]. Trap type was set as a fixed effect and sampling night as a random effect. As ecological data sets often include many ' 0 ' counts, models incorporated zeroinflation parameters, but this was found to have a nonsignificant effect on model fit and was not included as a parameter in the model. Tukey contrasts were used to compare differences in species abundance between trapping methods at $\alpha=0.05$.

\section{Anopheles species composition}

Binomial generalized linear models (GLMs) were employed in analysing the proportion of An. balabacensis caught in samples from different trapping methods. The proportion of anopheline samples that could not be identified were also analysed to investigate potential differences in sample quality between trapping methods. Tukey contrasts were used to compare differences between trap types.

\section{Associations between traps}

To assess the agreement in mosquito densities between methods baited with human hosts, Bland-Altman analysis was used [38]. This method provides a graphical representation of potential bias by showing the statistical limits of agreement, and confidence intervals of the mean difference between HLC and HENET, which indicates the magnitude of any systematic differences [39]. The difference between the two methods' nightly catch was plotted against the mean of their catch. Limits of agreement are considered to be met if $95 \%$ of differences fall within \pm 1.96 standard deviations of the mean difference. Nights on which neither method captured mosquitoes were excluded from analysis, as it is not possible to determine whether the zero count was attributable to lack of sensitivity in the methods, or the genuine absence of mosquitoes. Additional plots of differences between HLC and HENET as a percentage of their mean were also constructed to interrogate variability in differences under different mosquito densities. 


\section{Human participants}

Written informed consent was given by collectors performing HLCs and occupying tents for electrocuting net traps. All human participants were offered doxycycline as an anti-malarial prophylaxis and screened for malaria infection before the study commenced, half way through the study, and again at the end of their enrolment and 2 weeks after the experiment had ended. In the event that a participant showed signs of malaria, rapid assessment and medical treatment was provided at Hospital Kudat. Follow-up monitoring was also offered.

\section{Use of non-human primates}

The use of non-human primates in this study necessitated compliance with the Animals (Scientific Procedures) Act [40] Code of Practice for the Housing and Care of Animals Used in Scientific Procedures, guidelines set by the National Centre for the Replacement, Refinement and Reductions of Animals in Research [41], and under the authority of the Sabah Wildlife Department. Full details of animal husbandry and safety procedures can be found in Additional file 1 .

\section{Results}

Over 40 nights of outdoor trapping, a total of $5679 \mathrm{fe-}$ male mosquitoes were collected (Table 1). Specimens from 38 different species were caught. Culicine mosquitoes represented $90 \%$ of the overall catch and comprised 27 species (Table 2) and 584 anophelines representing 11 species made up the remaining 10\% (Table 3).

Combining across mosquito genera, a total of 31 mosquito species were recorded in HLCs compared to 22 in MBTs. Human and macaque-baited electrocuting nets detected eleven and seven species respectively. Focusing on anophelines, HLCs caught ten different species, HENETs seven and MENETs five. The MBT caught only one anopheline mosquito, An. latens. The most dominant Anopheles species in this study (out of 584 collected) were An. tessellatus (39.7\%), An. balabacensis (25.9\%) and An. subpictus (14.9\%).

Twenty three of the mosquito species sampled during collections have been implicated as vectors of diseases including malaria, dengue virus and lymphatic filariasis (Tables 2, 3). Of culicince species that could be

Table 1 Summary of mosquitoes caught by each trap type over a 40 night Latin square experiment in Kudat District, Malaysian Borneo

\begin{tabular}{llllll}
\hline Subfamily & HLC & MBT & HENET & MENET & Total \\
\hline Anophelinae & 403 & 1 & 146 & 34 & 584 \\
Culicinae & 741 & 369 & 3407 & 578 & 5095 \\
Total & 1144 & 370 & 3553 & 612 & 5679 \\
\hline
\end{tabular}

Abbreviations: HLC human landing catch, MBT monkey-baited trap, HENET human-baited electrocuting net, MENET monkey-baited electrocuting net identified, vector species included Culex vishnui (19.8\%), Aedes vexans (14.2\%), Cx. gelidus (14.0\%), and Cx. sitiens $(10.8 \%)$, which are all known vectors of Japanese encephalitis [42, 43] (Table 2). Culex gelidus is also a vector of Getah virus [44]. Aedes albopictus, implicated in dengue, chikungunya and Zika virus transmission, was also detected in both human (HLC, HENET) and monkey-baited traps (MENET).

Within the 11 anopheline species detected, six malaria vector species were identified (Table 3), of which An. balabacensis, An. latens and An. maculatus are regarded as significant primary malaria vectors, while $A n$. donaldi, $A n$. subpictus and An. tessellatus are considered secondary vectors with specific geographical areas $[25,26,45,46]$. With the exception of An. balabacensis and An. subpictus, all of these malaria vector species are also recognised as vectors of filarial nematodes, including Brugia malayia and Wuchereria bancrofti. All 584 Anopheles specimens underwent PCR analysis to test for the presence of malaria parasites but none were found to be positive. Although only 34 out of 584 captured Anopheles mosquitoes were bloodfed, representing less than $0.1 \%$ of the total catch, 97\% of these came from HLCs highlighting the inherent risk of exposure to mosquito bites during manual collections.

Across anopheline mosquitoes, species richness (Table 4) was greatest in the HLC, followed by the HENET then the MENET, and was lowest in the MBT and no further diversity analyses could be conducted on this method. However, the diversity indices for HLC and electrocuting net collections (both with humans and macaques) were very similar and clustered within 0.06 points of their diversity indices (range 0.64-0.69). The HLC and HENET had identical evenness indices (Table 4), suggesting a similar characterization of overall anopheline community diversity and relative species abundances. The MENET had a slightly higher Evenness index than either HENET or HLC, indicating that although anopheline diversity was lower in the MENET, those species that were attracted to macaque odours were caught in more similar numbers that those attracted to human odours.

Quantitative comparisons between traps focus on anopheline mosquitoes and in particular An. balabacensis and An. latens, due to their implicated role in $P$. knowlesi transmission regionally and elsewhere in Malaysia. Throughout the experiment, the MBT performed poorly for anopheline mosquitoes, only catching one anopheles specimen. Therefore, only limited analysis could be conducted for MBT results.

\section{Anopheles abundance}

The "gold standard" reference HLC method caught the greatest number of anopheline mosquitoes, with total catch descending in the order HLC $(n=403)$, HENET 
Table 2 Relative frequencies of culicine species caught by each trap type, and their medical significance as potential vectors of Japanese encephalitis (JE), chikungunya (CHKV), dengue (DENV) and Getah (GETV) viruses and filarial nematodes

\begin{tabular}{|c|c|c|c|c|c|c|}
\hline Species & Medical importance ${ }^{a}$ & HLC & MBT & HENET & MENET & Total \\
\hline Aedes albopictus & CHKV [49], DENV [50], JE [51] & 39 & 18 & 3 & 0 & 60 \\
\hline Ae. vexans & $J E[42]$ & 119 & 25 & 0 & 0 & 144 \\
\hline Armigeres species & & 1 & 0 & 0 & 0 & 1 \\
\hline Ar. jugraensis & & 0 & 2 & 0 & 0 & 2 \\
\hline Ar. malayi & & 0 & 1 & 0 & 0 & 1 \\
\hline Ar. moultoni & & 0 & 2 & 0 & 0 & 2 \\
\hline Ar. subabaltus & $J E[52]$ & 1 & 3 & 0 & 0 & 4 \\
\hline Ar. kesseli & & 2 & 0 & 0 & 0 & 2 \\
\hline Coquillettidia crassipes & Filariasis [53] & 7 & 9 & 1 & 0 & 17 \\
\hline Cq. ochracea & & 3 & 0 & 0 & 0 & 3 \\
\hline Culex species & & 48 & 0 & 1 & 0 & 49 \\
\hline Cx. bitaeniorhycus & $J E[42,51]$ & 0 & 1 & 0 & 0 & 1 \\
\hline Cx. fuscocephala & JE [43], GETV [44] & 7 & 1 & 0 & 0 & 8 \\
\hline Cx. gelidus & JE [43], GETV [44] & 39 & 102 & 0 & 1 & 142 \\
\hline Cx. malayi & & 0 & 1 & 0 & 0 & 1 \\
\hline Cx. mimules & & 0 & 1 & 0 & 0 & 1 \\
\hline Cx. perplexus & & 3 & 2 & 0 & 0 & 5 \\
\hline Cx. pseudovishnui & $J E[42]$ & 23 & 27 & 0 & 0 & 50 \\
\hline Cx. quinquefasciatus & JE [43], Filariasis [54] & 6 & 21 & 0 & 0 & 27 \\
\hline Cx. sitiens & $J E[51]$ & 96 & 13 & 0 & 0 & 109 \\
\hline Cx. tritaeniorhycus & $J E[43]$ & 35 & 24 & 1 & 0 & 60 \\
\hline Cx. vishnui & $J E[42,51]$ & 135 & 63 & 1 & 1 & 200 \\
\hline Cx. whitei & & 3 & 0 & 0 & 0 & 3 \\
\hline Cx. whitmorei & $J E[44]$ & 2 & 3 & 0 & 0 & 5 \\
\hline Mansonia species & & 5 & 0 & 0 & 0 & 5 \\
\hline M. annulata & Filariasis [55] & 2 & 0 & 0 & 0 & 2 \\
\hline M. annulifera & Filariasis [55] & 5 & 0 & 0 & 0 & 5 \\
\hline M. dives & Filariasis $[56,57]$ & 33 & 2 & 0 & 0 & 35 \\
\hline M. indiana & Filariasis [55] & 6 & 0 & 0 & 0 & 6 \\
\hline M. uniformis & Filariasis [55] & 59 & 3 & 0 & 0 & 62 \\
\hline Unidentified culicine & & 62 & 45 & 3400 & 576 & 4083 \\
\hline Total & & 741 & 369 & 3407 & 578 & 5095 \\
\hline
\end{tabular}

Abbreviations: HLC human landing catch, MBT monkey-baited trap, HENET human-baited electrocuting net, MENET monkey-baited electrocuting net ${ }^{\mathrm{a}}$ Reference number

$(n=146)$, MENET $(n=34)$, MBT $(n=1)$. Focussing on P. knowlesi vectors, An. balabacensis was collected by all methods except MBT; with a range in nightly abundance from 3.6 down to 0.2 per night (Fig. 2a). Human landing catches consistently caught more An. balabacensis than any other method (HLC $v s$ HENET: $z=6.20, P<0.001$; MENET: $z=-4.87, P<0.001$; Fig. 2a). However, there was no difference in mean nightly catch of An. balabacensis, between electrocuting nets baited with human or macaque odour $(z=-2.30, P=0.05$; Fig. $2 \mathrm{a})$. The number of $A n$. latens, another known malaria vector species, also differed significantly according to trap type ( $z=$ -4.27, $P<0.001)$, with the HLC again catching more than the HENET $(z=-2.73, P<0.05)$ and MBT $(z=$ -2.77, $P<0.01$; Fig. 2b). There was no different in An. latens nightly abundance between HENET and MENETs $(z=-0.71, P=0.7$; Fig. 2b).

\section{Representation of malaria vector species}

The proportion of An. balabacensis within the Anopheles mosquitoes collected varied between trap types (\% An. balabacensis, GLM, $z=-8.0, P<0.001$; Table 3). 
Table 3 Frequency of anopheline species caught by each trap type over 40 trapping nights, and their medical significance as potential vectors of human malaria (PHM, primary; SHM, secondary), simian malaria (SM) and the filarial nematodes Brugia malayi and Wuchereria bancrofti

\begin{tabular}{|c|c|c|c|c|c|c|}
\hline Species & Medical importance ${ }^{a}$ & HLC & MBT & HENET & MENET & Total \\
\hline An. balabacensis & $\begin{array}{l}\text { PHM [58], SM [59], } \\
\text { B. malayi [60], } \\
\text { W. bancrofti [60] }\end{array}$ & 162 & 0 & 12 & 1 & 175 \\
\hline An. barbumbrosus (s.l.) & & 6 & 0 & 7 & 5 & 18 \\
\hline An. donaldi & $\begin{array}{l}\text { SHM [61], } \\
\text { B. malayi [59] }\end{array}$ & 3 & 0 & 0 & 0 & 3 \\
\hline An. indefinitus & & 2 & 0 & 0 & 0 & 2 \\
\hline An. kochi & & 1 & 0 & 0 & 1 & 2 \\
\hline An. latens & $\begin{array}{l}\text { PHM [62], SM [14], } \\
\text { W. bancrofti [60] }\end{array}$ & 19 & 1 & 3 & 0 & 23 \\
\hline An. maculatus (s.l.) & $\begin{array}{l}\text { PHM [61], } \\
\text { W. bancrofti [61] }\end{array}$ & 14 & 0 & 1 & 0 & 15 \\
\hline An. peditaeniatus (s.l.) & & 1 & 0 & 0 & 0 & 1 \\
\hline An. subpictus (s.l.) & SHM [45] & 16 & 0 & 56 & 15 & 87 \\
\hline An. tessellatus (s.l.) & $\begin{array}{l}\text { SHM [61], } \\
\text { W. bancrofti [61] }\end{array}$ & 179 & 0 & 45 & 8 & 232 \\
\hline An. umbrosus (s.l.) & & 0 & 0 & 6 & 0 & 6 \\
\hline Unidentified anopheline & & 0 & 0 & 16 & 4 & 20 \\
\hline Total & & 403 & 1 & 146 & 34 & 584 \\
\hline
\end{tabular}

Abbreviations: HLC human landing catch, MBT monkey-baited trap, HENET human-baited electrocuting net, MENET monkey-baited electrocuting net Reference number

Anopheles balabacensis constituted a higher proportion of the Anopheles community in HLC collections $(40 \pm 2.4 \%)$ compared to the HENET $(8 \pm 2.3 \% ; z=6.3$, $P<0.001)$ and MENET collections $(3 \pm 2.9 \% ; z=-3.1$, $P<0.01$ ). The proportion of An. balabacensis did not vary significantly between electrocuting net traps baited with either humans or macaques $(z=-1.0, P=0.7)$. Anopheles latens constituted $5 \pm 1.1 \%$ and $2 \pm 1.2 \%$ of Anopheles caught in HLC and HENET samples respectively (no difference between methods, GLM, $z=1.3, P=0.4$ ), but this species was absent from MENET collections.

It was possible to identify $97 \%$ of anopheline samples to species using morphological features. All unidentified Anopheles samples came from electrocuting net traps. From these, $10 \pm 2.4 \%$ of anophelines in human and $9 \pm 4.4 \%$ in macaque-baited electrocuting net traps could not be identified to species, with no significant difference in the proportion of unidentifiable specimens between HENET and MENET (GLM, $z=-0.17, P=0.9$ ). Some diagnostic features such as scales and hairs were found to detach from specimens collected in electrocuting nets, which was the primary reason morphological identification was not possible. In contrast, only $20 \%$ of the culicine samples were identifiable on the basis of morphology, mostly from HLC and MBT. Culicine samples from electrocuting net trap samples were stored for longer than anophelines (in 70\% ethanol) prior to identification ( $>1$ year), and this may have resulted in greater loss of delicate diagnostic morphological features.

\section{Associations between HLC and HENET}

Simple correlation of catches from the HLC "gold standard" method and the alternative HENET method are shown in Additional file 3: Figure S1. In general, Bland-Altman plots indicated a consistency between the number of Anopheles caught in HLC and HENET samples (Fig. 3a, b), on the basis of $95 \%$ of nightly catches lying within the limits of agreement (Fig. 3a, dashed lines). At lower population densities (under mean catches of $\sim 15$ anophelines per night), the difference between nightly

Table 4 Anopheline species diversity indices by trap type

\begin{tabular}{lllll}
\hline Index & HLC & MBT & HENET & MENET \\
\hline Richness, $R$ & 10 & 1 & 7 & 5 \\
Gini-Simpson's Diversity Index, $1-D \pm 95 \% \mathrm{Cl}$ & $0.64 \pm 0.002$ & - & $0.69 \pm 0.007$ & $0.67 \pm 0.001$ \\
Simpson's Index of Evenness $E$ & 0.04 & - & 0.04 & 0.07 \\
\hline
\end{tabular}

Abbreviations: HLC human landing catch, MBT monkey-baited trap, HENET human-baited electrocuting net, MENET monkey-baited electrocuting net 


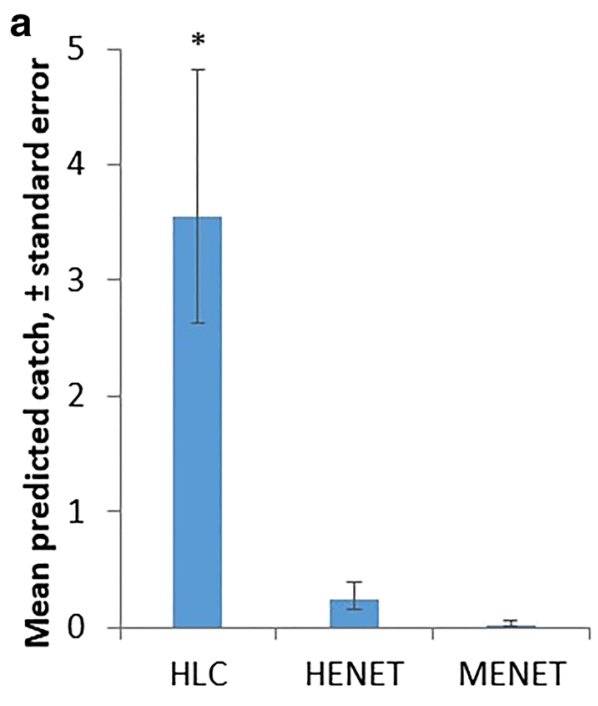

An. balabacensis

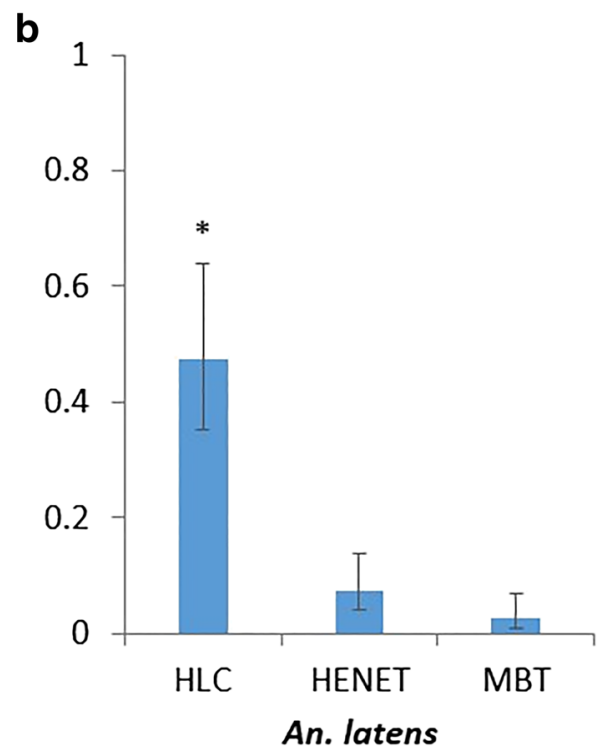

Fig. 2 Predicted mean nightly catches ( \pm standard error bars), of potential vectors of P. knowlesi; GLMM, P < $0.05\left(^{*}\right)$. a An. balabacensis. b An. latens. Abbreviations: HLC, human landing catch; MBT, monkey-baited trap; HENET, human-baited electrocuting net; MENET, monkey-baited electrocuting net

HLC and HENET catch is smaller, but increases as density increases. This apparent density-dependence implies that at higher mosquito densities, the HLC captures a greater number of anophelines than the HENET. The observed mean difference between the two methods (Fig. 3a, solid line) indicates that HLC caught an average of six to seven more individual anopheline mosquitoes each night than the HENET. This bias towards HLC is significant; the line of equality at 0 (Fig. 3a, dotted line) represents perfect agreement between the two methods (i.e. no difference in their catch), but falls outside of the observed mean difference and its envelope of $95 \%$ confidence intervals.

The density-dependence between HLC and HENET catches can be investigated further by plotting their mean catch against the difference in catch as a percentage of the mean (Fig. 3b). All data points lie well within the limits of agreement at \pm 1.96 standard deviations from the mean. The average difference between the two methods is $60.9 \%$ of the mean catch (Fig. 3b, solid line), although collections on nights with lower densities of anophelines show deviations of $\pm 200 \%$. Thus the relationship between the two methods is not consistent over the range of mean catches recorded.

\section{Discussion}

This study represents the first comparison between electrocuting nets and routine methods to sample mosquitoes attracted to human and macaque hosts. A wide range of mosquito species were sampled, encompassing a diverse array of vectors capable of transmitting both human and animal pathogens, including malaria, arboviruses and nematodes. Two recent studies conducted elsewhere in Sabah used the HLC method only to sample potential P. knowlesi vectors [47, 48]. Mosquito species composition differed somewhat between these studies, and with results reported here, indicating the huge diversity of human-biting mosquito fauna in this area of Malaysian Borneo and their ecological variability. However, a common finding is the presence of $A n$. balabacensis, the locally implicated vector of P. knowlesi, both in the present study and previous work [15].

Our key finding is that there is no one optimal sampling method that applies to all mosquito genera. Of the four sampling methods used here, the "gold standard" HLCs had the best performance for anophelines, including key malaria vector species in terms of overall abundance. However, electrocuting nets baited with either humans or macaque monkeys collected the higher number of culicine species, and also show promise for malaria vectors by catching Anopheles mosquitoes $(\sim 36 \%$ relative to HLC). In addition, there was accord between human-baited collection methods, the HLC and HENET, with respect to representative species diversity, which was similar in both. These two methods also shared identical evenness indices. Overall, electrocuting nets provide a standardised, relatively efficient means of sampling host-seeking mosquitoes, on a range of host types, and without exposing collectors to vector-borne diseases. Thus with further optimization to improve the condition of the specimens, they could be a valuable surveillance tool for vector-borne zoonses. One reason 

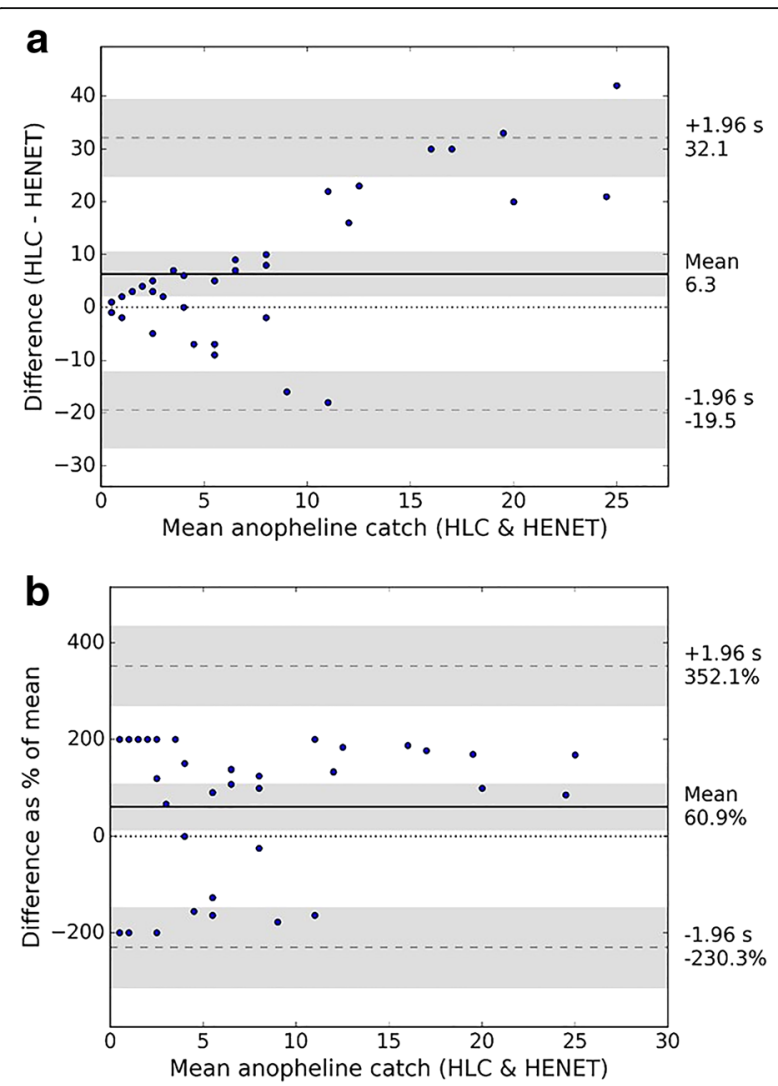

Fig. 3 Bland-Altman analysis of total Anopheles over 39 nights of catches from human landing catches $(\mathrm{HLC})$ and human-baited electrocuting nets (HENET). The line of equality (dotted line) represents perfect agreement between two methods. Mean difference (solid line) indicates bias from equality, limits of agreement are set at \pm 1.96 standard deviations of mean difference (dashed line, s), and both are shown with 95\% confidence intervals (shaded areas). a Mean vs difference. $\mathbf{b}$ Mean vs difference as a percentage of mean

the electrocuting nets performed less effectively than HLC could be that the plume of attractive odour released from tents may be reduced relative to the unprotected host, but on the basis of previous studies it is reasonable to assume that host odours were emitted [21]. In our experiment, we standardised traps baited with different animals by number of individuals, always using two humans versus two macaques, but standardizing by biomass is an alternative approach, which likely would have yielded quite different results. Our variant of the standard MBT performed worst for anophelines and also caught the lowest number of culicines.

All methods used in this study have limitations. A current weakness of electrocuting nets is that in contrast to other sampling methods around $10 \%$ of mosquito samples were damaged to the extent that they could not be identified morphologically. This percentage could perhaps be reduced by retuning the charge delivered by the grid and performing hourly collections from trays.
Alternative technology based on the same principles as traditional electrocuting nets is being developed using DC rather than AC power, which facilitates the use of much lower voltages and is associated with higher morphological identification rates in specimens [22, 23]. There is considerable scope to resolve these technical issues and thus provide a sampling method that does not have the ethical concerns associated with HLC.

In our study, monkey-baited traps did not perform particularly well as a sampling tool for anophelines. In previous studies using MBTs, mosquitoes were collected from within the net every $2 \mathrm{~h}$ [6] or periodically [7] during the nocturnal sampling period using manual or electrical aspirators. In consultation with Sabah Wildlife Department, neither of these collection methods were deemed appropriate to perform when macaques were in their enclosure, due to the risk to human health of repeated close contact (potential inhalation of viral particles via manual aspiration) and animal welfare (excessive noise from electrical aspirators). Therefore, collections were only undertaken at the end of the sampling night, once macaques had been removed. This may have resulted in opportunities for mosquitoes to escape the netting before being collected, accounting for the lower efficiency of MBTs here than reported previously [6, 7]. While comparisons of catches between MBTs are likely to remain a useful tool for understanding more about the ecology of macaque-biting mosquitoes [14], their precise design and operation may have to be modified to be fully compliant with current UK/EU guidelines for research involving non-human primates. Although we did not collect samples hourly during this study for electrocuting net traps, this would be feasible for both human and monkey-baited electrocuting nets, and would provide valuable data that is both comparable to hourly HLCs and important in characterising disease transmission and exposure risk.

Previous P. knowlesi vector studies in Southeast Asia have compared MBT catches with HLCs to draw inferences about zoonotic vector host preferences, such as using the ratio of individual mosquitoes from a given species in HLC to that in the MBT. For instance, Jiram et al. [7] calculated the ratio of the An. cracens from HLC to MBT to be 1:2.6, suggesting a greater preference for macaque hosts in this vector. However, HLC and MBTs differ greatly in the way in which they sample host-seeking mosquitoes, so potential methodological biases cannot be easily disentangled from real hostspecific differences in mosquito attraction. Electrocuting traps, on the other hand, use exactly the same means to sample mosquitoes attracted to host-associated odours, even when the animal producing the odours differs. When all experimental aspects of trapping methodology were standardised here, we found that An. balabacensis 
was caught at a ratio of 12:1 in human-baited to monkey-baited electrocuting nets, although the sample size was relatively low. This raises questions about the host specificity of this vector species; and thus the potential for zoonotic transmission of $P$. knowlesi parasites in Sabah.

\section{Conclusions}

Deciding which vector trapping technology to use in any given context will be a result of balancing technical and practical limitations, ethical issues, financial costs and the nature of required data. The urgent need to replace HLCs with a consistent, exposure-free method is compounded in the context of zoonotic and residual disease transmission settings by the requirement that any alternative method may also need to monitor mosquito activity on other animal hosts. Here we show the potential for electrocuting nets to address both these challenges in the context of zoonotic malaria transmission from wildlife reservoirs to humans. The expense and logistics required to set up electrocuting net traps (< USD 100/unit) makes them suited to long-term monitoring at sentinel sites and use in specific field studies on host-seeking behaviour. These initial costs are offset by the robust comparisons possible between different baits used in the trap, and the protection the method affords to bait animals. Standardised methods, such as electrocuting nets, should be developed further to ensure inferences in vector research are biologically, not methodologically, determined.

\section{Additional files}

Additional file 1: Text. Ethical considerations for the use of non-human primates. (DOCX $16 \mathrm{~kb}$ )

Additional file 2: Table S1. Description of primers used for detection of malaria parasite species in Anopheles mosquito specimens. (DOCX $47 \mathrm{~kb}$ )

Additional file 3: Figure S1. Correlation in catches of anopheline mosquitoes by human landing catch (HLC) and human-baited electrocuting net (HENET). (DOCX $15 \mathrm{~kb}$ )

\section{Abbreviations}

GLM: Generalized linear model; GLMM: Generalized linear mixed model; HENET: Human-baited electrocuting net; HLC: Human landing catch; MBT: Monkey-baited trap; MENET: Monkey-baited electrocuting net: PCR: Polymerase chain reaction; PPE: Personal protective equipment

\section{Acknowledgements}

The authors would like to thank EcoHealth Alliance, Sabah Wildlife Department, Danau Girang Field Centre and Dr. Milena Salgado-Lynn for providing guidance on health, safety and welfare aspects of working with non-human primates, and Sabah Wildlife Rescue Unit for macaque husbandry. We thank Matthew Stibbs for programming guidance, and Indra Vythilingam, Beth Downe, Sophie Richie, Albert Lim, Kimberley Fornance, Fazreen Mohd and the MONKEYBAR field team in Sabah for support throughout the study. We thank the community of Tajau Laut for their cooperation and interest during this research.

\section{Funding}

This research was funded by the Medical Research Council, Natural Environment Research Council, Economic and Social Research Council, and
Biotechnology and Biosciences Research Council through the Environmental and Social Ecology of Human Infectious Diseases Initiative, grant no. G1100796.

\section{Availability of data and materials}

The datasets supporting the conclusions of this article are included within the article and its additional files.

\section{Authors' contributions}

TC, CD, SJT and HMF conceived of the study. FH, BO, SJT, HMF and TC designed the experiments. $\mathrm{FH}, \mathrm{BO}$ and $\mathrm{TC}$ implemented the experiments and collected the data. BO carried out molecular analysis and $\mathrm{BO}$ and $\mathrm{SHN}$ identified samples. FH carried out statistical analysis. FH and HMF drafted the manuscript. All authors read and approved the final manuscript.

\section{Ethics approval and consent to participate}

This study was approved by the Malaysian National Medical Research Register (NMRR, Ref. NMRR-12-786-13,048) and the London School of Hygiene and Tropical Medicine Research Ethics Committee (REC, Ref. 6302) and Animal Welfare and Ethical Review Body (AWER, Ref. 2012/8 N). Consent was obtained from the village leader of Tajau Laut before data collection commenced.

\section{Consent for publication}

Not applicable.

\section{Competing interests}

The authors declare that they have no competing interests.

\section{Publisher's Note}

Springer Nature remains neutral with regard to jurisdictional claims in published maps and institutional affiliations.

\section{Author details}

'Agriculture Health \& Environment Department, Natural Resources Institute University of Greenwich, Central Avenue, Chatham, Kent ME4 4TB, UK. ${ }^{2}$ Department of Pathobiology and Medical Diagnostics, Faculty of Medicine and Health Sciences, Universiti Malaysia Sabah, Jalan UMS, 88400 Kota Kinabalu, Sabah, Malaysia. ${ }^{3}$ Department of Vector Biology, Liverpool School of Tropical Medicine, Pembroke Place, Liverpool L3 5QA, UK. ${ }^{4}$ Faculty of Infectious and Tropical Diseases, London School of Hygiene and Tropical Medicine, Keppel Street, London WC1E 7HT, UK. Institute of Biodiversity, Animal Health and Comparative Medicine, University of Glasgow, Glasgow G12 8QQ, UK.

Received: 27 March 2017 Accepted: 6 July 2017

Published online: 18 July 2017

References

1. World Health Organization. Manual on practical entomology in malaria. Part II. In: Methods and techniques. Geneva: World Health Organization; 1975.

2. Achee NL, Youngblood L, Bangs MJ, Lavery JV, James S. Considerations for the use of human participants in vector biology research: a toll for investigators and regulators. Vector Borne Zoonotic Dis. 2015;15(2):89-102.

3. Phyo AP, Nkhoma S, Stepniewska K, Ashley EA, Nair S, McGready R, et al. Emergence of artemisinin-resistant malaria on the western border of Thailand: a longitudinal study. Lancet. 2012;379:1960-6.

4. Tun KM, Imwong M, Lwin KM, Win AA, Hlaing TM, Hlaing T, et al. Spread of artemsinin-resistant Plasmodium falciparum in Myanmar: a cross-sectional survey of the K13 molecular marker. Lancet. 2015;15:415-21.

5. Wharton RH, Eyles DE, Warren MCW. The development of methods for trapping the vectors of monkey malaria. Ann Trop Med Parasitol. 1963;57: 32-46.

6. Vythilingam I, NoorAzian YM, Huat TC, Jiram Al, Yusri YM, Azahari AH, et al. Plasmodium knowlesi in humans, macaques and mosquitoes in peninsular Malaysia. Parasit Vectors. 2008; 1:26

7. Jiram Al, Vythilingam I, NoorAzian YM, Yusof YM, Azahari AH, Fong MY. Entomologic investigations of plasmodium knowlesi vectors in Kuala Lipis, Pahang, Malaysia. Malar J. 2012;11:213.

8. Silver JB. Mosquito ecology: field sampling methods. Third ed. Dordrecht: Springer Verlag; 2008. 
9. Stinton JA, Mulligan HW. A critical review of the literature relating to the identification of the malaria parasites recorded from monkeys of the families Cercopithecidae and Colobidae. Rec Malar Surv India. 1932;1II:381-443.

10. Singh B, Sung LK, Matusop A, Radhakrishnan A, Shamsul SSG, Cox-Singh J, et al. A large focus of naturally acquired Plasmodium knowlesi infections in human beings. Lancet. 2004:363:1017-24.

11. Vythilingam I. Plasmodium knowlesi in humans: a review on the role of vectors in Malaysia. Trop Biomed. 2010;27:1-12.

12. Rajahram GS, Barber BE, William T, Grigg MJ, Menon J, Yeo TW, Anstey NM Falling Plasmodium knowlesi malaria death rate among adults despite rising incidence, Sabah, Malaysia, 2010-2014. Emerg Infect Diseases. 2016;22(1):41-8.

13. Vythilingam I, Hii J. Simian malaria parasites: special emphasis on Plasmodium knowlesi and their Anopheles vectors in Southeast Asia. In: Manguin S, editor. Anopheles mosquitoes - new insights into malaria vectors. Online: InTech; 2013. p. 487-510.

14. Tan CH, Vythilingam I, Matusop A, Chan ST, Singh B. Bionomics of Anopheles latens in Kapit, Sarawak, Malaysian Borneo in relation to the transmission of zoonotic simian malaria parasite Plasmodium knowlesi. Malar J. 2008;7:52.

15. Wong ML, Chua TH, Leong CS, Khaw LT, Fornace K, Wan-Sulaiman W-Y, et al. Seasonal and spatial dynamics of the primary vector of Plasmodium knowlesi within a major transmission focus in Sabah, Malaysia. PLoS Negl Trop Dis. 2015;9(10):1-15

16. Torr SJ, Vale GA. Know your foe: lessons from the analysis of tsetse fly behaviour. Trends Parasitol. 2015;31(3):95-9.

17. Vale GA. New field methods for studying the responses of tsetse flies (Diptera, Glossinidae) to hosts. Bull Entomol Res. 1974;64:199-208.

18. Gillies MT, Jones MDR, Wilkes TJ. Evaluation of a new technique for recording the direction of flight of mosquitoes (Diptera: Culicidae) in the field. Bull Entomol Res. 1978;68:145-52.

19. Dugassa S, Lindh JM, Torr SJ, Oyieke F, Lindsay S, Fillinger U. Electric nets and sticky materials for analysing oviposition behaviour of gravid malaria vectors. Malar J. 2012;11:374.

20. Knols BGJ, Mboera LE, Takken W. Electric nets for studying odour-mediated host-seeking behaviour of mosquitoes. Med Vet Entomol. 1999;12:116-20.

21. Torr SJ, della Torre A, Calzetta M, Costantini C, Vale GA. Towards a fuller understanding of mosquito behaviour: use of electrocuting grids to compare the odour-orientated responses of Anopheles arabiensis and An. quadriannulatus in the field. Med Vet Entomol. 2008;22:93-108.

22. Maliti DV, Govella NJ, Killeen G, Mirzai N, Johnson PCD, Kreppel K, Ferguson HM. Development and evaluation of mosquito-electrocuting traps as alternatives to the human landing catch technique for sampling hostseeking malaria vectors. Malar J. 2015;14:502.

23. Govella NJ, Maliti DF, Mlwale AT, Masallu JP, Mirzai N, Johnson PCD, et al. An improved mosquito electrocuting trap that safely reproduces epidemiologically relevant metrics of mosquito human-feeding behaviours as determined by human landing catch. Malar J. 2016;15:465.

24. Barber BE, William T, Dhararaj P, Anderios F, Grigg M, Yeo TW, Anstey NM. Epidemiology of Plasmodium knowlesi malaria in north-east Sabah, Malaysia: family clusters and wide age-distribution. Malar J. 2012;11:401.

25. Sallum MAM, Peyton EL, Harrison BA, Wilkerson RC. Revision of the Leucosphyrus group of Anopheles (Cellia) (Diptera, Culicidae). Rev Bras Entomol. 2005;49(Suppl. 1):1-152.

26. Sallum MAM, Peyton EL, Wilkerson RC. Six new species of the Anopheles leucosphyrus group, reinterpretation of An. elegans and vector implications. Med Vet Entomol. 2005;19:158-99.

27. Rattanarithikul R, Harrison BA, Harbach RE, Panthusin P, Coleman RE. Illustrated key to the mosquitoes of Thailand IV. Anopheles. Southeast Asian J Trop Med Public Health. 2006;37:2

28. Phillips A, Simon C. Simple, efficient, and non-destructive DNA extraction protocols for arthropods. Ann Entomol Soc Am. 1995;88(3):281-3.

29. Singh B, Bobogare A, Cox-Singh J, Snounou G, Abdullah MS, Rahman HA. A genus- and species-specific nested polymerase chain reaction malaria detection assay for epidemiologic studies. Am J Trop Med Hyg. 1999;60(4):687-92.

30. R Core Team. R: a language and environment for statistical computing. Vienna: R Foundation for Statistical Computing; 2015.

31. Canty A, Ripley B. Boot: bootstrap R (S-plus) functions. In: R package version 1; 2016. p. 3-18.

32. Fournier DA, Skaug HJ, Ancheta J, lanelli J, Magnusson A, Maunder MN, et al. AD model builder: using automatic differentiation for statistical inference of highly parameterized complex nonlinear models. Optim Methods Softw. 2012;27:233-49.
33. Bates $D$, Maechler $M$, Bolker $B$, Walker S. Fitting linear mixed-effect models using Ime4. J Stat Softw. 2015;67(1):1-48.

34. Venables WN, Ripley BD. Modern applied statistics with S. Fourth ed. New York: Springer; 2002.

35. Hothorn T, Bretz F, Westfall P. Simultaneous inference in general parametric models. Biom J. 2008:50(3):346-63.

36. Peet RK. The measurement of species diversity. Annu Rev Entomol. 1974;5: 285-307.

37. Crawley MJ. Statistics: an introduction using R. West Sussex, England: John Wiley \& Sons; 2005.

38. Bland JM, Altman DG. Measuring agreement in method comparison studies. Stats Methods Med Res. 1999;8:135-60.

39. Giavarina D. Understanding bland-Altman analaysis. Biochem Med. 2015; 25(2):141-51.

40. Animals (Scientific Procedures) Act 1986. Available at: http://www.legislation. gov.uk/ukpga/1986/14/contents (Accessed 31 Oct 2016).

41. NC3Rs. Guidelines - primate accommodation, care and use. London: NC3Rs; 2006

42. Sucharit S, Surathin K, Shrestha SR. Vectors of Japanese encephalitis virus (JEV): species complexes of the vectors. Southeast Asian J Trop Med Public Health. 1989;20(4):611-21.

43. Vythilingam I, Oda K, Mahadevan S, Abdullah G, Thim CS, Hong CC, et al. Abundance, parity, and Japanese encephalitis virus infection of mosquitoes (Diptera: Culicidae) in Sepang District, Malaysia. J Med Entomol. 1997;34(3): 257-62.

44. Peiris JS, Amerasinghe PH, Amerasinghe FP, Calisher CH, Perera LP, Arunagiri CK, et al. Viruses isolated from mosquitoes collected in Sri Lanka. Am J Trop Med Hyg. 1994;51(2):154-61.

45. Reid JA. Anopheline mosquitoes of Malaya and Borneo. Stud Inst Med Res Malaya. 1968:31:520.

46. Harrison BA, Scanlon JE. Medical entomology studies II. The sugenus Anopheles in Thailand (Diptera: Culicidae). Contrib Am Entomol Inst. 1975;12(1):1-307.

47. Manin BO, Ferguson HM, Vythilingam I, Fornace K, William T, Torr SJ, et al. Investigating the contribution of peri-domestic transmission to risk of zoonotic malaria infection in humans. PLoS Negl Trop Dis. 2016;10:10.

48. Brant HL, Ewers RM, Vythilingam I, Drakeley C, Benedick S, Mumford JD. Vertical stratification of adult mosquitoes within a tropical rainforest in Sabah, Malaysia. Malar J. 2016:15:370.

49. Noridah O, Paranthaman V, Nayar SK, Masliza M, Ranjit K, Norizah I, et al. Outbreak of chikungunya due to virus of central/east African genotype in Malaysia. Med J Malaysia. 2007;62(4):323-8.

50. Singh KR, Paul SD. Isolation of dengue viruses in Aedes albopictus cell culture. Bull World Health Organ. 1969;40(6):982-3.

51. Vythilingam I, Oda K, Mahadevan S, Vijayamalar B, Morita K, Tsuchie H, Igarashi A, et al. Isolation of Japanese encephalitis virus from mosquitoes collected in Sabak Bernam, Selangor, Malaysia in 1992. J Am Mosq Control Assoc. 1995;11(1):94-8.

52. Muslim A, Fong M-Y, Mahmud R, Lau Y-L, Sivanandam S. Armigeres subalbatus incriminated as a vector of zoonotic Brugia pahangi filariasis in suburban Kuala Lumpur, peninsular Malaysia. Parasit Vectors. 2013;6:219.

53. Chiang GLCW, Samarawickrema WA, Mak JW, Kan SK. Filariasis in Bengkoka peninsula, Sabah, Malaysia: vector studies in relation to the transmission of filariasis. Southeast Asian J Trop Med Public Health. 1984;15(2):179-89.

54. Vythilingam I, Huat TC, Ahmad NW. Transmission potential of Wuchereria bancrofti by Culex quinquefasciatus in urban areas of Malaysia. Trop Biomed. 2005;22(1):83-5.

55. Wharton $\mathrm{RH}$. The biology of Mansonia mosquitoes in relation to the transmission of filariasis in Malaya. In: Bulletin of Institute for Medical Research Malaya; 1962.

56. Rubis P, Chang MS, Nagum AJ, Jau JL. Parasitological and entomological studies on filariasis in seven villages, Serian District, Sarawak, East Malaysia. Southeast Asian J Trop Med Public Health. 1981;12(1):30-5.

57. Chang MS, Chan KL, Ho BC. Control of Mansonia mosquitoes, vectors of brugian filariasis in Sarawak, Malaysia. Southeast Asian J Trop Med Public Health. 1993;24(Suppl. 2):93-104.

58. Peters W, Garnham PCC, Killick-Kendrick R, Rajapaksa N, Cheong WH, Cadigan FC. Malaria of the orangutan (Pongo pygmaeus) in Borneo. Phil Trans R Soc London. 1976;275:439-82.

59. Cheong WH, Warren M, Omar AH, Mahadevan S. Anopheles balabacensis identified as vector of simian malaria in Malaysia. Science. 1965;150(3701): 1314-5. 
60. Pokrovski VI, Nguyen TA, Godovannyi B. A filariasis in the developing countries of Southeast Asia. Meditsinskaya Parazitologiya i Parazitarnye Bolezni. 1976;4: $47-50$.

61. Harrison BA, Scanlon JE. Medical entomology studies II. The subgenus Anopheles in Thailand (Diptera: Culicidae). Contrib Am Entomol Inst. 1975;12:78.

62. White GB. The importance of Anopheles leucosphyrus group mosquitoes as vectors of malaria and filariasis in relation to transmigration and forestry in Indonesia with assessments of Anopheles balabacensis ecology and vectorial capacity. Jakarta: WHO Vector Biology and Control Research Unit; 1983.

Submit your next manuscript to BioMed Central and we will help you at every step:

- We accept pre-submission inquiries

- Our selector tool helps you to find the most relevant journal

- We provide round the clock customer support

- Convenient online submission

- Thorough peer review

- Inclusion in PubMed and all major indexing services

- Maximum visibility for your research

Submit your manuscript at www.biomedcentral.com/submit 